\title{
Immune control of an SIV challenge by a heterolgous and direct mucosal vaccination regimen in rhesus monkeys
}

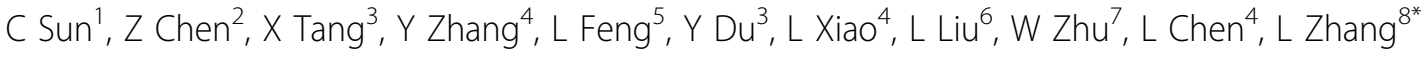 \\ From AIDS Vaccine 2012 \\ Boston, MA, USA. 9-12 September 2012
}

\section{Background}

The mucosal surface is the major route for HIV-1 transmission, yet a safe and effective AIDS vaccine through direct mucosal immunization remains elusive.

\section{Methods}

Here, we report a novel vaccination regimen consisting of a mucosal prime with replication-competent vaccinia Tiantan rMVTTSIVgpe and an intramuscular boost with non-replicating rAd5SIVgpe expressing SIV Gag, Pol and Env. Twenty Chinese rhesus macaques were used to evaluate its safety, immunogenicity and protective potential.

\section{Results}

Compared with three control groups, the rMVTTSIVgperAd5SIVgpe regimen elicited robust cellular immune responses with enhanced magnitude, sustainability and polyfunctionality, and higher titers of neutralizing antibodies against SIVmac1A11. Moreover, one rMVTTSIVgperAd5SIVgpe vaccinated animal was fully protected, while the rest demonstrated 1.74- $\log$ and 1.2-log reductions in peak and set-point viral loads upon intrarectal challenge with a high dose (5x105 TCID50/animal) of a pathogenic and neutralization-resistant SIVmac239. Importantly, the rMVTTSIVgpe-rAd5SIVgpe vaccinated animals remained healthy up to 850 days post-challenge, while the majority $(\sim 75 \%)$ of controls progressed to simian AIDS. The protective effect was found to correlate with SIV-specific CD8+ T cell ELIspot responses against Gag and Pol, but not Env.

${ }^{8}$ Comprehensive AIDS Research Center, Tsinghua University, Beijing, China Full list of author information is available at the end of the article

\section{Conclusion}

Our findings indicate that vaccine strategy engaging the mucosal surface from the beginning of vaccination may provide protective immunity against HIV-1 infection in humans.

\section{Author details}

${ }^{1}$ Guangzhou Institute of Biomedicine and Health, CAS, Guangzhou, China. ${ }^{2}$ AIDS Institute, LKS Faculty of Medicine, University of Hong Kong, China. ${ }^{3}$ AIDS Institute, LKS Faculty of Medicine, University of Hong Kong, China. ${ }^{4}$ Guangzhou Institute of Biomedicine and Health, CAS, China. ${ }^{5}$ Guangzhou Institute of Biomedicine and Health, CAS, China. ${ }^{6}$ AIDS Institute, LKS Faulty of Medicine, University of Hong Kong, China. ${ }^{7}$ AIDS Research Center, Chinese Academy of Medical Sciences, China. ${ }^{8}$ Comprehensive AIDS Research Center, Tsinghua University, Beijing, China.

Published: 13 September 2012

\section{doi:10.1186/1742-4690-9-S2-03}

Cite this article as: Sun et al:: Immune control of an SIV challenge by a heterolgous and direct mucosal vaccination regimen in rhesus monkeys. Retrovirology 2012 9(Suppl 2):03.

Submit your next manuscript to BioMed Central and take full advantage of:

- Convenient online submission

- Thorough peer review

- No space constraints or color figure charges

- Immediate publication on acceptance

- Inclusion in PubMed, CAS, Scopus and Google Scholar

- Research which is freely available for redistribution
(0) 2012 Sun et al; licensee BioMed Central Ltd. This is an Open Access article distributed under the terms of the Creative Commons Attribution License (http://creativecommons.org/licenses/by/2.0), which permits unrestricted use, distribution, and reproduction in any medium, provided the original work is properly cited. 